\title{
LINGUISTICA PRAGMATICA E STUDI CONTRASTIVI
}

1. È risaputo che la pragmalinguistica, al pari della sociolinguistica (con cui ha in comune molti temi) nasce per una specie di reazione allo strutturalismo saussuriano e al trasformazionalismo chomschiano. Trattasi comunque di una importante acquisizione della linguistica contemporanea che studia la lingua non in quanto sistema astratto, ma come strumento di comunicazione concretamente usato." "Comprendere e studiare l'uso effettivo del linguaggio, come di altri segni, è divenuto una esigenza pressante dei nostri giorni. “2

Nell'intricato labirinto delle teorie linguistiche moderne - per usare le parole di Giulio Lepschy ${ }^{3}$ - ci sembra in ogni caso molto convincente l'impostazione semiotica del Morris secondo cui noi, oltre alla fonologia, distinguiamo tre campi di investigazione linguistica, e precisamente il campo della pragmatica, quello della semantica e quello della sintassi. ${ }^{4}$ Invertendo l'ordine di questi tre componenti, come fa il De Mauro, ${ }^{5}$ possiamo dire che intendiamo pienamente una frase quando la valutazione sintattica (relativa ai rapporti funzionali dei componenti della frase tra loro) e semantica (relativa ai rapporti della frase con i loro possibili denotati) si integrano con la valutazione prammatica, relativa ai rapporti tra dictum e dicens, ossia tra segno e utente. Va notato che qui si parla solo di frasi, e che siamo quindi ancora fuori della sfera della linguistica testuale.

Usando ancora le parole del Morris nella bella versione di Silvio Ceccato, "se in un caso si fa esplicito riferimento a colui che parla $o$, esprimendoci in modo più generale, a chi usa un linguaggio, allora siamo nel campo della pragmatica. "6 Ed è a questo punto che va messo bene in rilievo come 1 ' informazione referenziale di un messaggio, oltre al significato denotativo, oggettivo, include spesso anche significati emotivi, affettivi, stilistici e connotativi in genere, in base ai quali va analizzata la pertinenza comunicativa dei singoli enunciati che possono anche oltrepassare $i$ limiti della proposizione. Siamo allora nel campo della linguistica testuale il cui recente avvento ha segnato uno sviluppo decisivo della struttura informativa del linguaggio,

\footnotetext{
Gaetano Berruto, La sociolinguistica, Bologna 1987, 4.

Charles Morris, Segni, linguaggio, comportamento, Milano 1963 (traduz. di Silvio Ceccato), 11.

nella prefazione a Ducrot-Todorov, o.c., XIII.

Morris, o.c., 211 (sulla scia di R. Carnap).

Tullio De Mauro, Introduzione alla semantica, Bari 1966, 151.

Morris, o.c., 211 (sempre citando R. Carnap).
} 
rendendo possibile l'applicazione di criteri più estensivi e più sistematici nelle analisi pragmalinguistiche. ${ }^{7}$

2. Dopo questa breve delineazione introduttiva, passiamo alla trattazione concreta del nostro problema. L'occasione ci viene offerta da una recente importante ricerca del nostro festeggiato, l'illustre italianista croato prof. Pavao Tekavčić, ricerca intitolata "Verso una pragmatica contrastiva delle cosiddette 'particelle' in serbocroato e in italiano". ${ }^{8}$ Trattasi di quelle forme particolari espressive che in determinati contesti perdono il loro significato di base assumendo funzioni comunicative speciali. Secondo le parole di Tekavčić, queste forme "non esprimono soltanto la sfera soggettiva del parlante e sintatticamente non tutte sono fuori di frase ${ }^{\text {". }}$. Una posizione, quindi, che si discosta in parte da una definizione largamente diffusa.

3. Già in precedenti pubblicazioni Pavao Tekavčić ci aveva fornito una casistica relativamente vasta dell'uso di queste forme, chiamate appunto 'particelle', termine in verità polivalente e che l'autore usa "faute de mieux", cioè in mancanza di una voce più appropriata che specifichi meglio queste forme espressive. Nel suo recente lavoro sopra citato, al quale faremo riferimento nel corso della nostra esposizione, il nostro autore constata innanzi tutto che le grammatiche croate e serbe trattano il problema delle 'particelle' in maniera insufficiente, mentre le grammatiche italiane non lo tratterebbero affatto. ${ }^{10}$ Le ricerche del Nostro gli hanno fruttato un inventario di 149 unità serbocroate di cui ben 94 rappresentano integrazioni originali sue in aggiunta alle sole 55 riscontrate nelle quattro grammatiche esaminate. ${ }^{11}$ Quanto alle 'particelle' italiane che secondo una tradizione linguistica non vengono trattate separatamente nelle grammatiche, ma vengono incluse tra le parti invariabili del discorso, massime tra gli avverbi, Tekavčić ne elenca una considerevole quantità nel corso del suo lavoro. Sono sempre ricerche compiute con fine intuito che interessano particolarmente da una visuale contrastiva e più precisamente nel campo della lessicografia bilingue. Nelle sue varie ricerche l'autore ci offre esempi interessanti di raffronto fra l'italiano e il serbocroato. In alcuni casi avremmo desiderato anche un tertium comparationis, praticamente il tedesco, come lingua egemone dell'Europa centrale, a cui l'autore ricorre solo sporadicamente nel corso della sua trattazione.

4. Prendiamo ad esempio la particella serbocroata "samo" che in origine ha significato restrittivo ${ }^{12}$ corrispondente all'italiano solo, soltanto, solamente. I vocabolari assegnano in ambedue le lingue due funzioni fondamentali a queste forme $\mathrm{e}^{13} \mathrm{e}$ così anche in tedesco:

7 Interessanti a tal proposito i lavori di K. Lichem e di M. Berretta.

8 É riportato il titolo del riassunto italiano dello studio (pag. 193). Il titolo originale è citato nella bibliografia in calce.

9 Tekavčić, o.c. (1989), 193.

10 Tekavčić, o.c., 129. Va detto comunque che i vocabolari più recenti (in particolare lo Zingarelli) trattano in maniera abbastanza diffusa i problemi dei significati connotativi delle parti invariabili del discorso, specie per gli avverbi.

11 Tre recenti grammatiche croate e una serba.

12 Il concetto di restrizione viene elaborato da $P$. Tekavčić in un ampio saggio del 1984.

13 Solo è comunque in primo luogo aggettivo. 


\section{Funzione avverbiale:}

Ulaznica stoji samo petsto dinara.

Il biglietto costa solo cinquecento dinari.

Die Eintrittskarte kostet nur fünfhundert Dinar.

2. Funzione di congiunzione:

Samo ne znam da li je to točno.

Soltanto non so se ciò sia esatto.

Nur weiss ich nicht ob das stimmt.

Dal significato originario, primitivo della forma samo si sono sviluppate in seguito alcune funzioni pragmatiche che vengono finemente analizzate dal nostro autore e tradotte in italiano con forme appropriate. Alla versione italiana abbiamo aggiunto anche qui e in seguito la nostra traduzione in tedesco. ${ }^{14}$

1) Significato esortativo:

Samo nastavite tako, mladicu, $i$ uspjeh je, osiguran!

\section{Reci ću sve tati! \\ - Samo reci, pa ceš vidjeti!}

\section{Nemoj mi samo reći da sve to nisi već prije znao.}
Kako ti samo pada na pamet tako nešto!

Gdje ste ga samo pronašli, kad nitko ne zna njegovu adresu?

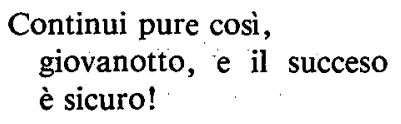
Fahren Sie nur so fort, mein Junge, und der Erfolg ist gesichert!

2) Lo stesso in senso ironico:

$$
\begin{aligned}
& \text { Dirò tutto a papà! } \\
& \text { - Diglielo pure, e } \\
& \text { vedrai! }
\end{aligned}
$$

Ich sage alles dem Papa!

- Sag es ihm nur, dann wirst du schon sehen!

3) Samo come rafforzamento di una proibizione:
Non dirmi soltanto (non dirmi poi) che tutto ciò non lo sapevi già prima.
Sag mir nur nicht dass du dies alles nicht schon früher gewusst hast.

4) Samo denotante un particolare interesse o meraviglia:

$$
\begin{aligned}
& \text { Come mai ti viene } \\
& \text { in mente qualcosa } \\
& \text { di (una cosa) simile! } \\
& \text { Dove mai lo avete } \\
& \text { trovato, se nessuno } \\
& \text { conosce il suo indirizzo? }
\end{aligned}
$$

14 I significati pragmatici di samo vengono trattati dal nostro autore nell'o.c. (1989), alle pagg. 145-146. 
A questi esempi di usi pragmatici della "particella" samo possiamo aggiungerne degli altri ${ }^{15}$ :

\author{
Kad bi samo došao! \\ Da samo mogu dobiti \\ taj novac prije \\ odlaska!
}
Idi se igrati, samo me pusti na miru!

Kako je samo došao ovamo?
5) In proposizioni "ottative":
Se solo venisse!
Wenn er nur käme!
Se potessi ricevere questo denaro prima della partenza!
Dass ich nur dieses Geld vor der Abreise bekäme!

6) In senso condizionale:
Va a giocare, basta che mi lasci in pace!
Geh spielen, nur lass mich in Ruhe!

7) In frasi interrogative (in aggiunta al num. 4):
Come ha fatto a venire qui?

Osservazione. - Come risulta da alcuni degli esempi citati, la "particella" $s a$ mo non viene sempre tradotta in italiano. Diversa è invece la situazione per il tedesco.

Nel grande Vocabolario della lingua croata o serba dell'Accademia di Zagabria, sotto la voce samo, definita come avverbio e congiunzione, vengono elencati costrutti che a volte si avvicinano semanticamente a quelli più sopra elencati.

5. Una tipica funzione pragmatica viene assunta talora dall'avverbio lijepo, derivato dall'aggettivo lijep (it. bello, ted. schön). Tekavčić ci dà innanzi tutto due esempi di uso di questo avverbio nel suo significato primario (nel quale può essere sostituito anche da altre forme, come fino, divno, krasno). ${ }^{15 a}$

1) Lijepo in funzione primaria:

Tata, položio sam
zadnji ispit.
- Lijepo, sine moj!

Tata, opet sam pao na ispitu.

- Lijepo, sine moj!

$$
\begin{aligned}
& \text { Papà, ho dato } \\
& \text { l'ultimo esame. } \\
& \text { - Bene, figlio mio! }
\end{aligned}
$$

2) Lijepo in senso ironico:
Papà, sono stato bocciato di nuovo all'esame.
- Ma bravo, figlio mio!

\section{Papa, ich habe die letzte Prüfung abgelegt. - Schön, mein Sohn!}
Papa, ich bin wieder durchgefallen bei der Prüfung.
- Schön, mein Sohn!16

15 v. la nota 20.

15a Tekavčić, o.c. (1989), 147.

16 In ambedue gli esempi le versioni, italiana e tedesca, sono nostre. 
Per quanto in italiano esista l'avverbio bellamente, esso negli esempi sopra citati sarebbe fuori posto, come bene osserva il nostro autore. Perciò egli propone in questi casi locuzioni del tipo che bello!, questa sì che è bella! (quest' ultima locuzione specie in senso ironico).

I significati pragmatici dell'avverbio lijepo si riscontrano soprattutto nel linguaggio colloquiale per indicare partecipazione, affabilità, impegno, ironia. Tekavčić ci offre alcuni esempi di questo uso e osserva che per l'italiano in questi casi si deve ricorrere ad altri mezzi espressivi, qui compresa l'intonazione. Possiamo aggiungere che nella versione in un'altra lingua la forma alle volte può venir tralasciata. Ecco gli esempi citati dall'autore e rimasti introdotti. Le versioni sono nostre in tutti e tre $\mathrm{i}$ casi:

\section{...Vi lijepo sjednite $k$ peći... a ja ču lijepo prigledati radi prilike za Jalševo. ${ }^{17}$}

Sad se najprije lijepo raskomoti i odmori, a o poslu ćemo razgovarati kasnije.

Gle ti njega! Čovjek lijepo strugnuo na more $i$ ostavio nama cijeli posao.
Lei si sieda con comodo accanto alla stufa... e io cercherò di informarmi sul modo migliore di raggiungere Jalševo.

Accomodati innanzi tutto e riposati per bene, poi parleremo dei nostri affari.

Ma guarda un po' che tipo! Lui bel bello se ne va al mare e addossa a noi tutto il lavoro.
Setzen Sie sich schön neben den Ofen und ich werde trachten eine Möglichkeit zu finden dass Sie Jalševo erreichen.

Jetzt mache dich vor allem schön bequem und ruhe dich aus, dann werden wir über unsere Geschäfte sprechen.

Sieh da den Kerl! Er macht sich schön davon, geht ans Meer und überlässt uns die ganze Arbeit!

Nelle nostre versioni, quello che interessa è in primo luogo come è resa la "particella" lijepo. Ecco comunque alcuni altri esempi di uso di questa "particella":

Lijepo ga pozdravi.

Lijepo smo se udesili.

Ostavi ti to lijepo na miru.

Molim lijepo!
Salutalo caramente.

Stiamo freschi.

Guardati bene dal fare qualcosa.

Prego! Per favore!
Lass ihn schön grüssen.

Da sind wir schön dran.

Lass das schön bleiben.

Bitte schön!

Significati pragmatici dell'avverbio lijepo sono presenti già presso scrittori croati e serbi dei secoli passati. Ne abbiamo la documentazione nel grande vocabolario storico dell'Accademia di Zagabria (v. il vol. VI alle pagine 75-77). Nella lingua colloquiale sembra che questo uso sia stato secondato, specie presso i bilingui del se-

17 Questo passo che Tekavčić ha tratto da un romanzo dello scrittore croato August Senoa (1838-1881) viene riferito qui in forma abbreviata. 
colo $\mathrm{XIX}^{\circ}$ e anche in seguito, dalla frequente presenza della forma tedesca schön. Nella versione italiana, invece, lijepo viene spesso tradotto con altri mezzi o tralasciato.

6. L'avverbio već (it. già, ted. schon) nel suo significato originario indica che un'azione o un fatto, nel momento al quale ci si riferisce, è compiuto o sta compiendosi: tutto è già preparato; il sole sta già tramontando. L'avverbio in tutte e tre le lingue può riferirsi anche a un avvenimento che si prevede nel futuro: fra poco sarà già di ritorno ${ }^{18}$.

Il primo uso pragmatico della particella već che l'autore sottopone a vaglio è quello di servire da motivo di convincimento ossia di persuasione nei confronti dell'interlocutore:

Ne uzrujavaj se, saznat ce on to već na vrijeme.

Ne bojte se, stvar će se već dobro završiti.

Platiti će oni to već jednog dana!
Non eccitarti, egli verrà a saperlo in tempo.

Niente paura, la cosa finirà bene.

Vedrai che lo pagheranno un giorno!19
Rege dich nicht auf, er wird es schon rechtzeitig erfahren.

Nur keine Angst, die Sache wird schon ein gutes Ende nehmen.

Sie werden es schon eines Tages bezahlen!

In un secondo uso pragmatico di questa particella si allude a una situazione nota che serve da sostegno per una conclusione logica:

Mira, kako su već ženske brbljave, ni tog puta nije znala sačuvati tajnu.
Mira, da chiacchierone come sono le donne, nemmeno quella volta seppe serbare il segreto.
Mira, wie die Frauen schon geschwätzig sind, konnte auch diesmal das Geheimnis nicht hüten.

La particella već preceduta da un imperativo e seguita dall'avverbio jednom, esprime, secondo un'altra fine analisi di P. Tekavčić, un'anticipazione affettiva di una conclusione desiderata. Molto confacente la versione italiana dell'autore in cui viene auspicata la "volta buona".

Ta prestani već jednom! Smettila una buona volta! So hör' schon einmal auf!

18 cfr. Zingarelli, Dizionario, sotto la voce già.

19 Varianti proposte da Tekavčić: "Sono certo che lo pagheranno un giorno. Verrà il giorno che lo pagheranno. Lo pagheranno. Lo pagheranno un giorno! “ 
Ecco alcuni esempi aggiuntivi:20

On ce ti to već reci.

Već radi ljudi.

Kad je već tako sposoban, morao bi raditi bolje.

Već sama pomisao na to me uznemiruje.
Vedrai che te lo dirà.

Se non altro per la gente.

Giacché è così bravo, dovrebbe lavorare meglio.

Solo a pensarci mi agito.
Er wird es dir schon sagen.

Schon wegen der Leute.

Wenn er schon so tüchtig ist, müsste er besser arbeiten.

Schon der Gedanke daran macht mich unruhig.

Osservazione. - La particella tedesca schon in molti casi viene tralasciata sia in italiano che in croato o serbo:

Ich komme schon!

Es ist schon recht, aber es könnte besser sein.
Vengo!

Sto venendo!

Va bene, ma potrebbe essere meglio.
Dolazim!

Upravo dolazim!

Dobro je, ali bi moglo biti bolje.

7. Nel suo ampio elenco di "particelle" pragmatiche Tekavčić annovera anche l'avverbio inače (it. se no, altrimenti; ted. sonst). Pero Budmani, il secondo redattore in ordine di tempo del grande Vocabolario storico del croato o serbo, e in seguito Petar Skok fanno derivare questo avverbio dall'aggettivo protoslavo inak (lat. diversus). Tekavčić considera la forma inače un elemento profrastico, sostituto di una proposizione, che rimanda anaforicamente a un contesto precedente. P. es.:

\section{Moraš završiti zadaću, inače . Devi finire il compito, se no večeras ne ideš u kino. stasera non vai al cinema. \\ Du musst die Aufgabe been- den, sonst gehst du heute Abend nicht ins Kino.}

Il nostro autore ci offre anche la parafrasi del significato di questo elemento profrastico: "Se si realizza il contrario del fatto in questione". ${ }^{21}$.

Quanto ai significati secondari, pragmatici della particella inače l'autore cita questo esempio:
A kako je inače?
E per il resto, come va?
Und sonst?
$A$ inače, kako je?
Wie geht es?

\footnotetext{
20 Per le versioni in tedesco dei singoli esempi di questo contributo siamo ricorsi in parte al Dizionario Sansoni citato in calce. - Sarà compito degli storici del serbocroato di stabilire se le equivalenze col tedesco risalgano a influssi diretti o mediati, p.es., al ceco, o siano dovuti, almeno in parte, a poligenesi.

21 Ecco il testo originale (o.c., pag. 169): “Ako se ostvari suprotna mogućnost od onoga o čemu je prije bilo govora".
} 
Per parte nostra citeremo alcuni esempi aggiuntivi:

Je li inače još nešto nejasno?

Tko drugi može inače to učitini?

Inače svugdje.

Inače ne preuzimam takve dužnosti.

Drukčije nego inače.

Tko inače?

Kao inače.
Oltre a ciò, c'è ancora qualcosa che non è chiaro?

Chi altri può

farlo?

In tutti gli altri luoghi.

Normalmente (in genere) non assumo tali incarichi.

Diverso del solito. Diversamente del solito.

Chi altri?

Come al solito. Come sempre.
Ist sonst noch etwas unklar?

Wer sonst kann das machen?

Sonst überall.

Sonst übernehme ich nicht solche Aufträge.

Anders als sonst.

Wer sonst?

Wie sonst.

8. Tra le particelle con funzione pragmatica rientrano anche le interiezioni, e P. Tekavčić ce ne offre parecchi esempi che interessano da una visuale contrastiva. Così, in corrispondenza alla forma del croato o serbo $p a^{22}$ risponde il modello italiano be':

Je li taj liječnik sposoban? $P a$, jest.

\section{È bravo questo medico? \\ Be', sì. ${ }^{23}$}

Il significato pragmatico-connotativo di questo be' seguito da una pausa, evidenziata dalla virgola, esprime un certo tentennamento nella risposta, la quale è bensì confermativa, include però un significato tra scettico e offensivo.

In un altro esempio ${ }^{24}$ la particella be' (cs. dakle) assume una funzione articolatoria di segnale d'inizio di una conversazione, ${ }^{25}$ con cui l'ascoltatore o gli ascoltatori vengono invitati a seguire la comunicazione del parlante:
Dakle, što mi se danas dogodilo, to valj- da do smrti neću više doživjeti.

Be', quello che mi è successo oggi non mi accadrà probabilmente più in tutta la vita.

Bruno Migliorini nel suo Vocabolario della lingua italiana (Torino 1965) elaborando i significati della voce bene vi inserisce anche la seguente osservazione di carattere pragmatico: "Come introduzione al discorso: Bene, via, ditemi cos'avete; e per troncarlo: Bene, via, contentatevi. In questi casi si tronca fam. in Be'. "Consul-

22 Nei vocabolari generalmente registrata solo come congiunzione.

23 Tekavčić, o.c., 152.

24 Tekavčić, o.c., 166-167.

25 In tedesco: "Gliederungssignal". Cfr. K. Lichem. Bemerkungen zu den Gliederungssignalen im gesprochenen Italienisch sta in Ch. Schwarze, Italienische Sprachwissenschaft, Tübingen 1981, 61-82 (citazione da P. Tekavčić). 
tando vocabolari più recenti abbiamo potuto accertare che in essi i vari significati pragmatici di questa interiezione vengono vagliati in maniera più completa. Tuttavia siamo ancor lontani da esemplificazioni che soddisfino, per cui dobbiamo dar ragione a Giovanni Nencioni quando afferma:

"È risaputo lo scarso interesse che i grammatici e i lessicografi hanno sempre portato alle interiezioni. I primi hanno spesso dubitato di inserirle a pieno titolo nella eletta schiera delle 'parti del discorso', i secondi le hanno lematizzate e definite raramente e scarsamente... " ${ }^{26}$

Già lo Spitzer nella sua ben nota "Italienische Umgangssprache" (Lingua colloquiale italiana) ${ }^{27}$ aveva lamentato questo disinteresse della linguistica per le interiezioni e, basandosi su opere di teatro e dizionari, aveva cercato di chiarire i tratti fondamentali della funzione dialogica delle interiezioni nel parlato, studiando particolarmente le forme di apertura e di chiusura del discorso. ${ }^{28}$ L'elemento psicologico nel suo metodo di analisi sarebbe stato influenzato da Bally, come afferma l'autore stesso nella prefazione del suo studio. ${ }^{29}$

E qui è il caso di ricordar brevemente la classificazione abbastanza diffusa delle interiezioni in due gruppi: 1) quelle monosillabiche come $a h, e h, o h, m a h, b e h, p o h$, tutte polisemiche e parafrasabili in vario modo, e 2) quelle "improprie" costituite da sostantivi, aggettivi e altre parti del discorso usate come interiezioni: coraggio!, peccato!, bravo!, bene!, ecco!, presto!, viva!, basta!, figuriamoci! e simili, tutte forme a forte quoziente semantico, notevolmente influenzabili nella loro funzione espressiva dall'intonazione.

Rientrano in questa serie anche forme come vedi, senti, guarda, bene analizzate da Patrizia Manili in uno studio del 1983. ${ }^{30}$ Trattasi di forme che hanno perso del tutto il loro rapporto con il significato semantico di base e servono a richiamare l'attenzione dell'ascoltatore sul discorso che segue. ${ }^{31}$ Particolare interesse suscitano le varie parafrasi di certe forme, come quelle esclamative del tipo guarda un po'?, ma senti!, che denotano "la meraviglia del parlante di fronte all'apprendimento di un fatto che viene a tradire le sue aspettative." ${ }^{\text {"32 }}$ Anche la dettagliata analisi della forma polivalente magari con tutte le sue evoluzioni semantiche, presentata da Maria Grazia Spiti ${ }^{33}$ ci interessa in questa sede tanto più che include rapporti semantici

26 Nencioni, o.c. in calce, 233.

27 Bonn u. Leipzig 1922, pp. XX + 313.

28 Il primo capitolo del libro tratta appunto delle "Eröffnungsformen des Gesprächs", mentre l'ultimo, il quarto, tratta delle "Abschlussformen".

29 Da ricordare, vent'anni dopo, l'importante contributo di S. Karcevski, Introduction à l'étude de l'inlerjection, in "Cahiers F. de Saussure“, 1, 1941 (citato da G. Nencioni). - Ch. Bally, che già nel 1905 aveva pubblicato il suo Précis de stylistique tornerà sull'argomento delle interiezioni in seguito.

3o Manili, o.c. in calce.

31 Manili, o.c., 11.

32 Manili, o.c., 15 .

33 Spiti, o.c. in calce. 
con la forma croata o serba makar (di origine discussa). Ma è un problema che richiederebbe un discorso a parte.

Tornando alle interiezioni monosillabiche del tipo $a h, e h$, oh va detto che rappresentano alle volte problemi per i traduttori raffigurando anch'esse, come le interiezioni improprie, vere parole frasi, spesso semanticamente dubbie. La loro resa in un codice diverso può quindi diventare complicata e faticosa. ${ }^{34}$ Lo dimostrano alcuni begli esempi riportati da Giovanni Nencioni nel suo studio citato, dove alle volte un $e h$ ? viene parafrasato con "Siamo intesi, vero?" o può equivalere a "Non è cosi ?"35 Un caso estremo l'autore ce lo porge riproducendo una scena dal Piacere dell'onestà di Pirandello: "Maur./.../. Ha qualche debito. Fabio. Quanti? Molti? Oh, me l'immagino!“. Qui, secondo l'ingegnosa parafrasi di Nencioni l'oh corrisponde a "non può essere altrimenti, dopo quanto mi hai detto e considerata l'azione che si presta a compiere. “36 In questi casi, come pure con i cosiddetti monoremi del tipo Orribile!, Maledizione!, Aiuto!, Coraggio! "la predicatività è dovuta all'intonazione, cioè all'intenzione comunicativa che si manifesta in essa ${ }^{\text {c37 }}$; osservazione molto acuta che si potrebbe forse completare con una piccola aggiunta precisativa: "dove il valore semantico della predicatività è dovuto all'intonazione..." Importante è comunque il fatto che l'autore ha messo in questi casi in evidenza il concetto di predicatività, quell'entità alle volte nascosta e misteriosa, onnipresente nelle varie specie degli enunciati umani e quindi presente anche nelle interiezioni che abbiamo tentato di esaminare a conclusione della nostra noterella pragmalinguistica.

\section{OPERE CONSULTATE E CITATE}

Berretta, M., Connettivi testuali in italiano e pianificazione del discorso, in Linguistica testuale, Atti del XV Congresso intern. di studi della S.L.I., Roma 1984, pp. 237-254.

Berruto, G., La sociolinguistica, Bologna, Zanichelli 1987 , pp. VIII +159 .

Carnap, R., Introduction to Semantics, Cambridge 1942.

Dardano, M. e Trifone, P., La lingua italiana, Bologna, Zanichelli 1985.

De Mauro, T., Introduzione alla semantica, Bari Laterza 1966.

Ducrot, O. e Todorov, Tz., Dizionario enciclopedico delle scienze del linguaggio. Edizione italiana a cura di Giovanni Caravaggi, Milano 1972.

Jakobson, R., Saggi di linguistica generale a cura di Luigi Heilmann. Milano, Feltrinelli 1966.

Jernej, J., Riflessioni sulle unità linguistiche chiamate "particelle", in Italica Belgradensia 1990/3, Belgrado 1990, pp. 1-4.

4 Jakobson, o.c. in calce, 58.

35 Nencioni, o.c., 46.

36 . Nencioni, o.c., 253.

${ }^{37}$ Nencioni, o.c., 257. 
Lichem, K., Connettivi e demarcativi. Aspetti diacronici preliminari in Linguistica storica e cambiamento linguistico, Atti del XVI Congr. intern. di studi della S.L.I. (Firenze, 7-9 maggio 1972), Roma 1985, pp. 211-225.

Manili, P., Per un'indagine su vedi, senti, guarda (e forme collegate), Perugia, Le edizioni universitarie 1983, pp. 86.

Morris, Ch., Segni, linguaggio, comportamento. Traduzione dall' originale inglese di Silvio Ceccato. Milano, Longanesi 1963, pp. 338.

Nencioni, G., L'interiezione nel dialogo teatrale di Pirandello, in "Studi di grammatica italiana“, VI (1977), pp. 227-263.

Spiti, M.G., "Magari“ tra scritto e parlato, in Gli annali dell'Università per Stranieri, Perugia 1986, pp. 133-163.

Spitzer, L., Italienische Umgangssprache, Bonn-Leipzig 1922, pp. XX +313 .

Tekavčić, P., Prema kontrastivnoj pragmatici tvz. "čestica" u hrvatskom ili srpskom jeziku, RAD JAZU, knj. 427, Zagreb 1989, pp. 127-194.

\section{DIZIONARI}

Dizionario delle lingue italiana e tedesca I/II, realizzato dal Centro lessicografico Sansoni sotto la direzione di Vladimiro Macchi, Firenze - Roma - Wiesbaden 1982.

Il nuovo Zingarelli, Bologna, Zanichelli 1983 (undicesima edizione).

Deanović M. e Jernej J., Vocabolario croato o serbo - italiano I/II Zagabria $1990^{\circ}$.

Rječnik hrvatskoga ili srpskoga jezika. Na svijet izdaje Jugoslovenska Akademija znanosti $i$ umjetnosti I/XXVI, Zagreb 1881-1976.

Wahrig, G., Deutsches Wörterbuch, Gütersloh 1968.

Sažetak

PRAGMATIČNA LINGVISTIKA I KONTRASTIVNE STUDIJE

Nakon teoretskog uvoda analiziraju se u članku pragmatična značenja nekih često upotrebljenih "čestica" kao što su samo, lijepo, već, inače, i to tragom temeljnje rasprave Pavla Tekavčića iz god. 1989. Dodani su i njemački prijevodi hrvatskim primjerima što su u spomenutoj raspravi prevedeni na talijanski. Članak završava napomenama koji se odnose na inače zapostavljenu kategoriju uzvika i njihova moguća pragmatična značenja. 